\title{
JÄGER ODER JAGUAR - AMBIVALENTE PERSPEKTIVITÄT UND HYBRIDE VERSPRACHLICHUNG ZU DER ERZÄHLUNG MEU TIO O IAUARETÊ UND IHRER ÜBERSETZUNG MEIN ONKEL DER JAGUAR ${ }^{1}$
}

\author{
Orlando Grossegesse ${ }^{1}$ \\ -Universidade do Minho, Braga, Portugal
}

\begin{abstract}
Resumo: No caso de textos ficcionais que encenam a perspetiva narrativa através de falas regionalmente específicas, o tradutor literário está confrontado com dificuldades quase insuperáveis. A oralidade que se constrói no contínuo de diálogo (unilateral) e fluxo de consciência constitui mais do que um problema de reprodução. A questão central é a perspetiva narrativa e as propostas de identificação dirigidas ao leitor implícito. No texto-alvo, isto se torna um desafio peculiar, não só para outra língua mas também para outro leitor, no caso de a constelação linguística binária ficar atravessada por uma terceira língua. É o caso da situação linguística híbrida na narrativa Meu tio o Iauaretê (1961) de João Guimarães Rosa traduzida para alemão por Curt Meyer-Clason em Mein Onkel der Jaguar (1981). A nossa abordagem parte da categoria da fala de proximidade (Ágel \& Hennig), tendo também em conta o processo autoreflexivo do tradutor que se manifesta no pósfácio e no glossário.

Palavras-Chave: João Guimarães Rosa; Curt Meyer-Clason; Fala de proximidade; Tupi / nheengatu
\end{abstract}

\footnotetext{
${ }^{1}$ Erstmals präsentiert auf der internationalen Konferenz Proximity and Distance in Language and Culture (3. bis 4. Dezember 2009, UCP, Lissabon), jedoch nicht publiziert in M. Franco \& B. Sieberg. Proximity and Distance in Language and Culture. Lisboa: Universidade Católica (Serie ECC), 2011. Der Beitrag wurde ins Deutsche übersetzt, überarbeitet und bibliographisch aktualisiert.
} 


\title{
JÄGER ODER JAGUAR - AMBIVALENT PERSPECTIVITY AND HYBRID LIFE. ON THE STORY MEU TIO O IAUARETE AND ITS TRANSLATION MY UNCLE THE JAGUAR
}

\begin{abstract}
Fictional texts which enact narrative perspective through regional specific speeches confront the literary translator with almost insuperable difficulties. The orality, constructed in the flux of unilateral dialogue and stream of conscience, is more than a problem of reproduction: the essential aspect is the perspective and the identification(s) offered to the implicit reader. In the target text this means a peculiar challenge, not only for a different language but also for a different reader, if a binary linguistic constellation is thwarted by a third language. This can be shown in the case of the hybrid linguistic situation in João Guimarães Rosa's Meu tio o Iauaretê (1961) in comparison with its translation into German by Curt-Meyer Clason in Mein Onkel der Jaguar (1981). Our approach is guided by the category of speech of immediacy (Ágel \& Hennig), taking also into account the translator's self-reflexive process as manifest in epilogue and glossary.
\end{abstract}

Keywords: João Guimarães Rosa; Curt Meyer-Clason; Speech of immediacy; Tupi / nheengatu

"Im heutigen Brasilien muß man weit reisen, um einen Jaguar aus der Nähe zu erleben; Guimarães Rosa verschafft einem dieses Erlebnis mit geradezu beängstigender Intuitivkraft”. Dieses Zitat aus einer Rezension der Stuttgarter Zeitung ziert den Buchdeckel der Taschenbuch-Ausgabe von Mein Onkel der Jaguar (Köln: KiWi, 1994). Im gleichen Jahr erschien die letzte Übersetzung, die Curt Meyer-Clason von Guimarães Rosas Werk machte: Tutaméia, unter Mitarbeit von Horst Nitschak. Die Rolle des Übersetzers als "Zwillingsbruder" des Autors, die Meyer-Clason mit seiner durch eigenes Brasilien-Erleben eingefühlten Transkreation von Grande Sertão (1964) begründet ${ }^{2}$, scheint bei diesen späteren Übersetzungen

\footnotetext{
${ }^{2}$ Siehe Meyer-Clason im Interview: "Eu tinha convivido com a terra dele. Assim é que consegui conviver com a obra dele, transformando-me no irmão gêmeo dele para escrever um novo livro na minha língua e no espírito dele." (Chiappini 370).
} 
weniger $\mathrm{zu}$ wirken. Stattdessen artikuliert sich ernüchterndes Bewußtsein der Begrenztheit angesichts des "grenzenlos[en]" Fächers der Ausdrucksmittel bei Guimarães Rosa, der allerdings bereits die Schreibweise von Grande Sertão bestimmt. Die evolutive Sichtweise, die Meyer-Clason im Nachwort von Tutaméia einnimmt, verschleiert dies. ${ }^{3}$ Der Text Meu tio o Iauaretê, erstmals 1961 veröffentlicht ${ }^{4}$, ist gar ein Nebenprodukt der Genese des großen Epos - beide charakterisiert der Monodialog. Im Falle der Erzählung kommt noch hinzu, dass eine Mensch-Tier-Verwandlung und in der Schlusssequenz das Sterben in dieser spezifischen Form direkter Rede im Dialog mit einem Gegenüber, dessen Rede nicht erscheint, präsentiert wird. Im Nachwort der Übersetzung (erstmals 1981 veröffentlicht) beantwortet Curt Meyer-Clason die Frage "wie kann Mein Onkel der Jaguar in einer fremden Sprache rezipiert werden?" (Meyer-Clason 79) folgendermaßen:

\begin{abstract}
Wahrscheinlich nur in einer zweiten Realität, und das heißt wörtlich: im übertragenen Sinn. Denn selten tritt das Problem des Unveräußerlichen, das jeder Sprache eigen ist, die Frage nach dem Nicht-Übermittelbaren, dem im Wortsinn Unübersetzbaren, deutlicher zutage als im vorliegenden Fall, zumal hier der Kern der Erzählung, die Triade Jaguar-TupiIndio, im Spiele ist, auf dem Spiele steht. (Meyer-Clason 79)
\end{abstract}

Diese "Praxis des Nachschöpfens" als krönende Phase seiner brasilianischen Lehrzeit ist laut Meyer-Clasons “Autofiktion der Übersetzerschaft" eng mit Guimarães Rosa verbunden (Grossegesse 117).

3 "In Tutaméia ist Rosas Sprache im Vergleich zu den früheren Werken noch komplizierter geworden. Nun verdreht er Sinn und Form der Wörter, verändert Redensarten, zerbricht den konventionellen Satzbau und versucht mit seinen Erfindungen die Ausdrucksmittel grenzenlos zu erweitern" (Meyer-Clason 261). Zur Übersetzung von Tutaméia und zur "Mitarbeit" von Horst Nitschak siehe Studie und Interview von Glica Machado Seidinger.

${ }^{4}$ In der Zeitschrift Senhor, Nr. 25 (1961) und danach posthum in Estas estórias (Rio de Janeiro: José Olympio, 1969). Hier zitiert nach der Ausgabe Rio de Janeiro: Nova Fronteira, 1985, pp. 160-198. 
Dieser tiefe Zweifel an der Übermittelbarkeit widerspricht einem "Erlebnis mit geradezu beängstigender Intuitivkraft" in der zu Beginn zitierten Rezension aus der Stuttgarter Zeitung. Ein solches wäre doch demjenigen des brasilianischen Lesers ähnlich, "der diese Geschichte ohne Einführung und Erläuterungen intuitiv, fast möchte man sagen: körperlich, nachzuvollziehen vermag" (MeyerClason 78-79). Bei dieser Formulierung erhebt sich der Verdacht der Idealisierung. Man fühlt sich an Meyer-Clasons frühere Definition (1967) der Brasilianer als "im Urzustand von Erde, Flora und Fauna verwurzelt" und daher "aus der Wahrheit der fünf Sinne» bestehend" erinnert (Meyer-Clason 17). Diese Verklärung essenzieller brasilidade markiert im Nachwort von Mein Onkel, der Jaguar den Gegenpol zum Leser der deutschen Übersetzung, an den eine zweifache Anweisung ergeht. "Je nach Temperament oder Neigung" könne er als Einstieg zwischen (1) unbewusster, intuitiver oder (2) bewusster Lektüre wählen. Erstere besteht darin "den Text unmittelbar auf sich wirken [zu] lassen, bezaubert oder befremdet" und ist für ein (rationalisierendes) Verständnis danach ergänzungsbedürftig. Letztere definiert Meyer-Clason im Gegensatz zu derjenigen "des Brasilianers im Sinne C. G. Jungs" als eine "distanziert-kritische, nicht mythisch-mystische Teilnahme" (MeyerClason 80). Im ersten Lektüreweg wird suggeriert, dass mythischmystische Teilnahme für den Leser der Übersetzung sowieso nur begrenzt möglich wäre - während für jeden Brasilianer ein durch Tupisierung und kreative Neuschöpfung zweifach verfremdeter Text problemlos, weil intuitiv erschließbar sei.

Das Fremde im Ausgangstext wird somit homogenisiert, obgleich sein hybrider Charakter vor allem für diejenigen Brasilianer, die nicht mit dem nheengatu, der supraethnischen Variante des Tupi im Kontakt mit dem Brasilianischen und anderen Indiosprachen $^{5}$, vertraut sind, zweifellos ein Hindernis darstellt.

$5 \mathrm{Ab}$ dem späten 19.Jahrhundert setzt sich der Begriff nheengatu (nheen = Sprache / Sprechen + gatu $=$ gut) durch. Es entstehen Verschriftlichungen (nheengatu / brasiliansich), Grammatik (Pedro Luis Simpson, 1877), Wörterbuch 
Dies wird stellenweise durch die Abfolge von Tupi-Begriff und seiner brasilianischen Übersetzung oder kontextuelle Einbettung gemildert. Doch trotzdem haben Bemühungen, die Lektüre ausgehend von Haroldo de Campos' Befund der Tupinização durch ein Glossar zu erleichtern (Perrone; Ávila \& Trevisan), nicht bloß akademisches Interesse. Zweifellos sind die unübersetzten TupiWorte in der Übersetzung stärker als etwas Fremdes markiert, denn im Gegensatz zum Brasilianischen steht das Deutsche nicht im Kontakt mit dem nheengatu. Auch gerundische Hybridformen wie "munhamunhando" (Unsinn redend) bleiben in der Zielsprache Fremdkörper. Jedoch konstituiert dies lediglich einen graduellen Unterschied gegenüber dem ebenfalls sprachlich heterogenen Ausgangstext. Für beide gilt zudem die Verfremdung durch Neuschöpfung auf Tupi-Basis, die bis in Lautreihungen übergehen kann. Dies ist fiktionsimmanent besonders motiviert durch die zeitweise Verwandlung des Mestizen in einen Jaguar. Der zentrale Begriff des Übergangs ist "jaguanhenhém”, ein Tupi-Neologismus für das Sprechen des Jaguars, der eine mythisierte Ureinheit von "gente-onça" wachruft.

In Meyer-Clasons Essenzialisierung kultureller Prädisposition wird der Identität des nheengatu die Autonomie genommen und per se partiell animalisiert zu einem "Tupi-Mischmasch" in doppelter Abhängigkeit, nämlich von der "brasilianischen Menschensprache" und von der "Tiersprache des Tupigeschöpfs Jaguar" (MeyerClason 78). Das von ihm benannte "Problem des Unveräußerlichen, das jeder Sprache eigen ist" (Meyer-Clason 79), bleibt implizit auf das Sprachpaar Brasilianisch / Deutsch bezogen; die nicht sehr aussagekräftige “Triade Jaguar-Tupi-Indio" (Meyer-Clason 79) bleibt auf das Erzählte bezogen, geschieden vom Diskurs in der "mineiro"-Variante des Brasilianischen", der doch mit dem

(Ermano Stradelli, 1929). Da dieser Aufsatz keine lingustische Studie ist, wird auch weiterhin der undifferenzierte Begriff Tupi bzw. Tupisierung verwendet.

${ }^{6}$ Meyer-Clason spricht vage von "Regionalismen portugiesischer und brasilianischer Prägung” (Meyer-Clason 77).

Cad. Trad., Florianópolis, v. 41, no 3 p. 395-410, set-dez, 2021. 
nheengatu über lexikalische Anleihen hinaus morphologisch und sogar syntaktisch kreativ amalgamiert wird, wie Ávila \& Trevisan (303-308) anschaulich zeigen. Dieses Amalgam gilt es nicht nur zu übersetzen, sondern gleichzeitig als solches spürbar zu machen.

Gegenüber Meyer-Clasons Selbstkommentar - ab jetzt nur noch sporadisch kontrastiv eingeblendet - sollen ausgehend vom Befund der graduell unterschiedlichen Heterogenität von Ausgangsund Zieltext die komplexen Verhältnisse in der Erzählung $\mathrm{Meu}$ tio o Iauaretê und in ihrer Übersetzung Mein Onkel der Jaguar beschrieben werden. ${ }^{7}$ Dabei dient die Theorie des Nähe- und Distanzsprechens (Ágel \& Hennig) als Bezugspunkt. Denn gerade fiktionale Texte, die Nähesprechen über regional spezifische oder charakteristische Sprechweisen inszenieren, stellen den literarischen Übersetzer vor schier unüberwindliche Schwierigkeiten angesichts der Unmöglichkeit, ein äquivalentes Nähesprechen in der Zielsprache zu schaffen. Alle Versuche in dieser Richtung müssen künstlich bleiben, da sie die kulturelle Distanz nicht naturalisieren können. Letztlich muß sich die Übersetzung auf die diskursiven Markierungen der Oralität beschränken ${ }^{8}$, die zwar vom Leser als solche verstanden, aber nicht als Nähesprechen empfunden werden. Diese Markierungen sind im Falle von Mein Onkel der Jaguar das Weglassen des Personalpronomens (des Sprechers und des Angesprochenen) und Verkürzungen (Elisionen; Verschmelzung von Verb und Pronomen), ohne das Hochdeutsche dialektal zu durchsetzen. Abweichungen wie die doppelte Negation "Damit ich nie mehr keine Angst bekomme!" (Rosa/CMC 18; "Pra eu nunca eu não ter medo!", Rosa 166) sind ebenso selten wie lautlich

\footnotetext{
7 "Rosa" identifiziert den Ausgangstext, "Rosa/CMC" die Übersetzung durch Curt Meyer-Clason. Eine weitergehende Übersetzungsanalyse würde den Rahmen dieses Aufsatzes sprengen. Dabei ist der vergleichende Blick auf Übertragungen in andere Sprachen (siehe Oseki-Déprés Vergleich von drei Übersetzungen ins Französische) unabdingbar.

${ }^{8}$ Siehe die Überlegungen in Faria \& Hatje-Faggion zum Problem der Mündlichkeit im literarischen Text (analysiert in den Übersetzungen von John Steinbecks Of Mice and Men).
} 
begründete Transkreation: "drum drum Bum! Knall und Fall... (Rosa/CMC 15; "por isso, por isso, pum! - porro de fogo...", Rosa 165). Die Besonderheit dieser Oralität ist ihr sprachlich hybrider Charakter, der im Zuge diskursiver Markierung in der Übersetzung ebenfalls reduziert wird. Beispielsweise sind in den Sequenzen über den Revolver "caipora", "panema" und "marupiara" (Rosa 191) ersetzt durch "Pech(strähne)" und "Dusel" (Rosa/CMC 61-62). Trotz dieser Enttupisierung sorgen noch reichlich TupiEinsprengsel für ein Befremden, das nur im aktiven Leseprozess zu einem Sich-Hineinversetzen in Nähesprechen trotz kultureller Distanz überwunden werden kann. Dies betrifft Ausgangs- und Zieltext in gradueller Weise und abhängig von der Prädisposition des jeweiligen Lesers.

Die perspektivische Textpragmatik gibt dem impliziten Leser Identifikationsangebote: dieser findet sich meist im ortsfremden verirrten Besucher präfiguriert ${ }^{9}$, an den sich der Sprecher teils mit periegetischem, teils mit narrativem Duktus richtet. Der Jaguarjäger gewährt Zuflucht - als Gegenleistung erhält er cachaça (durchgängig mit Schnaps naturalisiert), möchte zuweilen mehr ergattern; zugleich jagt er durch Narrative des Tötens und der Interpretation akustischer Wahrnehmungen von draußen seinem Gegenüber Furcht ein. Mit beiden Gesten stellt er seine Machtposition in seiner Hütte und im Terrain zur Schau, gipfelnd und endend in der Verwandlung, die zugleich eine definitive stimmliche ist: von Mischsprache zu nheengatu, das sich seinerseits in unterbrochene Lautfolge auflöst. Es überlagern sich zwei Transformationen, von Jäger zu Jaguar und von Leben zu Tod, wodurch auch Grenzsituationen des Ich-Sprechens kumulieren: sprachlich und existientiell.

Ein Vergleich der Anfangs- und Schlusssequenzen (1 und 2) in Ausgangs- und Zieltext soll die Aspekte exemplarisch verdeutlichen:

\footnotetext{
${ }^{9}$ Minderheitlich wäre an einen Leser mit primärer Sprachidentität nheengatu zu denken.
}

Cad. Trad., Florianópolis, v. 41, no 3 p. 395-410, set-dez, 2021. 
Hum? Eh-eh... É. Nhor sim. Ã-hã, quer entrar, pode entrar... Hum, hum. Mecê sabia que eu moro aqui? Como é que sabia? Hum-hum... Eh. Nhor não, n't, n't... Cavalo seu é esse só? Ixe! Cavalo tá manco, aguado. Presta mais não. Axi... Pois sim. Hum, hum. Mecê enxergou este foguinho meu, de longe? É. A'pois. Mecê entra, cê pode ficar aqui. (Rosa 160)

Hum? Eh-eh... Ja. Doch, Nhor. Aha, Sie wollen reinkommen, kommen Sie rein... Hum, hum. Sie wußten, daß ich hier wohne? Woher wußten Sie das? Humhum... Eh. Nein, Nhor, n't n't... Ihr einziges Pferd, das? Ixe! Das Pferd lahmt, hat Wasser, taugt nicht mehr. Axi... Natürlich, hum, hum. Haben wohl von weitem mein Feuerchen gesehen, wie? Ja. Na gut. Kommen Sie rein, Sie können hier bleiben. (Rosa/CMC 7)

Desvira esse revólver! Mecê brinca não, vira o revólver pra outra banda... Mexo não, tou quieto, quieto... Ói: cê quer me matar, ui? Tira, tira revólver pra lá! Mecê tá doente, mecê tá variando... Veio me prender? Ói: tou pondo mão no chão é por nada, não, é à-toa... Ói o frio... Mecê tá doido?! Atiê! Sai pra fora, rancho é meu, xô! Atimbora! Mecê me mata, camarada vem, manda prender mecê... Onça vem, Maria-Maria, come mecê... Onça meu parente... Ei, por causa do preto? Matei preto não, tava contando bobagem... Ói a onça! Ui, ui, mecê é bom, faz isso comigo não, me mata não... Eu - Macuncozo... Faz isso não, faz não... Nhenhenhém... Heeé!... Hé... Aar-rrâ... Aaâh... Cê me arrhoôu... Remuaci... Rêiucàanacê... Araaã...Uhm... Ui... Ui...Uh... uh... êeêê... êê... ê... ê... (Rosa 198) 
Weg mit dem Revolver! Spielen Sie nicht damit, drehen Sie ihn weg... Ich rühr mich ja nicht, bin ganz still, still... Ói: wollen Sie mich töten, ui? Werfen Sie den Revolver weg, weg damit! Sie sind krank, Sie werden ganz anders... sind Sie gekommen, um mich festzunehmen? Ói: ich leg meine Hand wegen nichts so auf den Boden, nur so... Ói, die Kälte... Sind Sie verrückt?! Atiê! Hinaus mit Ihnen, die Hütte gehört mir, xó! Atimbora! Sie töten mich, Ihr Kamerad kommt, der läßt Sie festnehmen... Jaguar kommt, Maria-Maria, frißt Sie... Jaguar ist mein Verwandter... Ei, wegen dem Schwarzen? Hab den Schwarzen nicht getötet, hab Unsinn erzählt... Ói, der Jaguar! Ui, ui, Sie sind gut, tun Sie das nicht mit mir, töten Sie mich nicht... Ich Macuncôzo...Tun Sie das nicht, tun Sie's nicht... Nhenhenhém... Heeé!... Hé... Aar-rrâ... Aaâh... Sie haben mich arrhoôu... Remuaci... Rêiucàanacê... Arraã ...Uhm... Ui... Ui...Uh uh êêêe. $\hat{e} \hat{e} \ldots \hat{e}_{\ldots} . . \hat{e} \ldots($ Rosa/CMC 72-73)

Die im Kontinuum von (einseitigem) Dialog und Gedankenrede konstruierte narrative Oralität, im russischen Formalismus als skaz bezeichnet, bedeutet also nicht ein auf Lexik beschränktes Übersetzungsproblem, so wie dies das Nachwort suggeriert, wenn es beispielsweise um die Schwierigkeit geht, die Vielfalt der Anredeformen des Sprechers an den Unbekannten zu übersetzen (Meyer-Clason 79-80). Vielmehr soll der implizite Leser durch Nähesprache Anreize erfahren, die in der Schrift konstruierte Mündlichkeit selbst stimmlich und körperlich zu performieren im Sinne von Paul Zumthor (1990). ${ }^{10}$ Dabei sind der Alkoholkonsum, der dem Sprecher die Zunge löst, und das Fieber, das den Besucher schläfrig und sprachfaul macht, fiktionsimmanent zu berücksichtigen.

Abgesehen vom Titel - bereits in der Ich-Perspektive der Rede - wird auf die Markierung von Schriftlichkeit verzichtet. Typische

\footnotetext{
${ }^{10}$ In diese Richtung argumentiert Thais Calvi Tait (2008) auf der Basis von Wolfgang Iser und Paul Zumthor. Im Nachwort lediglich definiert für den brasilianischen Leser (Meyer-Clason 78-79).
}

Cad. Trad., Florianópolis, v. 41, no 3 p. 395-410, set-dez, 2021. 
morpho-syntaktische Strukturen des Distanzsprechens fehlen. Das Nähesprechen erscheint unvermittelt mit seinen Interjektionen, Reihungen, Wiederholungen, Ellipsen, die graphisch in Auslassungszeichen und Gedankenstrichen enden. Was bleibt, ist die Materialität des Schriftmediums und die ihr zugeordnete normale Rezeptionssituation des stillen Lesens, die es zur Performanz des mündlichen Kontinuums aufzubrechen gilt. Allerdings wird dies im Falle der Übersetzung erschwert, da sie unübersetzte Elemente meist - nicht immer (siehe: Nhor) - durch Kursivsetzungen als Fremdes hervorhebt, jedoch keine Anweisung über die Aussprache der in portugiesischer Graphie erscheinenden Laute (vor allem $c$, $n h$ und die Nasalierung von Vokalen mit Tilde) gibt. Da die Ebene der Bedeutung dem deutschsprachigen Leser nur sehr begrenzt intuitiv bis kontextuell erschließbar ist, erschöpft sich das Erlebnis von kulturell ferner Nähesprache bald. Wieviele Leser brechen die Lektüre $a b$ ? Oder diese alterniert - sich weiter von Performanz entfernend - mit dem Konsultieren von Nachwort und Glossar. Der Übersetzer selbst hat diesen Zugang als distanziert-kritisch dem intuitiven gegenübergestellt (Meyer-Clason 80). Bleibt zu fragen, wie konsequent sich Übersetzung und Glossar ergänzen, um eine derartige Lektüre zu stützen und die Bedeutungsfülle in Meu tio o Iauaretê zu erschließen.

Global gesehen bleibt festzuhalten: es gibt keine Korrelation zwischen Kursivdruck und Glossar. Die Einträge entbehren jeglicher Systematik und folgen keinen einheitlichen Kriterien: hier erscheinen gängige brasilianische Begriffe wie Quiabo ${ }^{11}$, Umgangssprache (z.B. Namensverkürzungen Quim und Tiaguim ${ }^{12}$ )

11 Auch die kursive Hervorhebung von "quiabo" im Text ist unnötig (Rosa/CMC 30). Die Bezeichnung, die aus dem quimbundo stammt, erscheint im Glossar (Rosa/ CMC 84) mit einer Beschreibung und kulinarischem Hinweis ohne den im Deutschen gängigen Begriff “Okra”, der einer westafrikanischen Sprache entlehnt ist (wikipediaEintrag < https://de.wikipedia.org/wiki/Okra > . Version 25. Juni 2021).

${ }^{12}$ Auf -im endende Substantivformen sind typisch für "mineiro". Dagegen verdienen die zahlreich auftretenden tupisierten Eigennamen (siehe Ávila \& Trevisan 306-307) keinen einzigen Eintrag. 
ebenso wie Ausdrücke in nheengatu, die allerdings nur ein schmales Segment des bereits partiell enttupisierten Textes abdecken. Das Glossar huldigt eher João Guimarães Rosas Schöpferkraft ${ }^{13}$, als dem Leser morphologisch erläuterte Einträge an die Hand zu geben. So ist es beispielsweise in der Sequenz (1) falsch, das Ixe gemäß der Erklärung "entspricht dem brasilianischen $\mathrm{eu}=$ ich" (Rosa/CMC 81) zu entschlüsseln. Es ist zu vermuten, dass Guimarães Rosa gar mit der möglichen Verwechslung der fast homophonen Wörtern ixe (Interjektion, die Ironie oder Spott ausdrückt) und ixé (Personalpronomen) spielt (Ávila \& Trevisan 322). Bei der Sequenz (2) ist der Leser mit den Erklärungen zu den Wortmontagen "Rêiucàanacê" und "Remuaci" überfordert" ${ }^{14}$, da das Glossar nicht die agglutinativen Strukturen des nheengatu wie Prä- und Suffixe sowie die charakteristische Verdoppelung thematisiert, die übrigens auch das "mineiro"-Brasilianisch des Mestizen prägt. Konkret fehlt hier ein Eintrag "jucá” bzw. “jucajucá”, denn gerade dieses Verb mit der Bedeutung töten, in der Verdoppelung viel oder hintereinander töten (Ávila \& Trevisan 322-323), ist für den Text zentral. So heißt es etwa wenige Seiten vor Schluss: "Eh, juca-jucá, atiê, atiuca!" (Rosa 196; Rosa/CMC 70). Im Glossar findet sich "atiê", aber nicht das hier reimende "atiuca” (Interjektion, die Mitleid ausdrückt).

"Dem deutschen Leser das vollständige Instrumentarium von Rosas ,Tupisierung' seiner Erzählsprache in Mein Onkel zu liefern, würde den Rahmen einer literarischen Edition übersteigen”, heißt es im Nachwort (Meyer-Clason 78). Wie bereits bei Grande Sertão zieht Meyer-Clason die Grenze zwischen dem Wissenschaftler und dem Übersetzer, der dem Autor nachschöpft. Weit davon entfernt, Vollständigkeit des Glossars zu fordern, fehlen allerdings Einträge,

13 Wiederholt heißt es "Rosas Erfindung", "Wortmontage Rosas" und am ausführlichsten zu "Macuncôzo" als "Wortverschmelzung Rosas", mit einem längeren Briefzitat des Autors an Haroldo de Campos (Rosa/CMC 81-84).

${ }^{14}$ Siehe demgegenüber "Os últimos rugidos" (Ávila \& Trevisan 315-317). Hinzu treten Druckfehler, sowohl im Text als auch im Glossar: hier "Rêiuncàanacê" (Rosa/CMC 84) statt richtig "Rêiucàanacê".

Cad. Trad., Florianópolis, v. 41, no 3 p. 395-410, set-dez, 2021. 
die wesentliche Bedeutungen des Textes und damit die zwischen den Identitäten von Jäger und Jaguar changierende Nähesprache besser erschließen könnten. Dies soll an zwei Aspekten erläutert werden.

Die Kursivsetzung in folgendem Satz fällt aus dem Rahmen: "Und dann rief mich Nhô Nhuão Guede her, zum Enttigern" (Rosa/CMC 49). Das ist natürlich kein Tupi-Wort, sondern ein deutscher Neologismus für "desonçar"15, der den in Südamerika nicht heimischen Tiger einspielt. Verständlicherweise fehlt im Deutschen eine nähesprachliche Differenzierung von Raubkatzen, die gerade für die Lebenswelt von Meu tio o Iauaretê im Mittelpunkt steht. Doch die dem Habitat ferne Lösung mit Tiger verbaut den begrifflichen Kern der ambivalenten Perspektivität zwischen Jäger, der damit beauftragt wird, das Gebiet von Raubkatzen zu säubern "desonçar", und seine Transformation in eine Raubkatze - "onçar". Überhaupt bietet die Übersetzung keine von Jaguar verschiedene Begrifflichkeit für die Wortfamilie "onça", die doch den gesamten Diskurs des Mestizen variantenreich durchzieht. Das bedeutet nicht bloße sprachliche Verarmung, sondern den Abbau von Strukturen innerhalb der übergreifenden Isotopie von "onça". So bleibt die für das Nähesprechen zentrale Opposition zwischen "iauaretê" (der echte Jaguar) und "suaçurana" (die falsche Pumakatze), beide durch die Tupi-Suffixe -ête und -rana entsprechend markiert, konturlos. ${ }^{16}$ Im Text sorgt die Gleichsetzung von "Tiger" und "Jaguaretê" (Rosa/CMC 53) für weitere Verwirrung. Dabei ist das Ich doch in dem identitären Dilemma gefangen, als Raubkatzen-Jäger auch seine Jaguar-Verwandten zu jagen. Durch eine Fülle individualisierter Körper- und Verhaltensmerkmale und dadurch motivierter sprechender Namen (die das Glossar nur im Fall von "Canguçu” erklärt) erscheinen sie als dem Ich am nächsten im Gegensatz zu den verhaßten namenlosen "suaçurana", die lediglich physisch

15 “Então Nhô Nhuão Guede me mandou vir pra cá, pra desonçar.” (Rosa 184) ${ }^{16}$ Im Glossar erscheinen zwei Einträge "Jaguar" und "Jaguaretê", ohne die Bedeutungsnuancierung zu erklären (Rosa/CMC 82). Ein Eintrag zu "suaçurana" fehlt.

Cad. Trad., Florianópolis, v. 41, no 3 p. 395-410, set-dez, 2021. 
differenziert werden. In dem durch Doppelung intensivierten Namen der geliebten Maria-Maria gipfelt der anthroponyme Übergang individualisierter Zoonymie. Dies läßt den sich steigernden Hang, vom Jäger zum Jaguar zu mutieren, als einen natürlichen, nächstliegenden erscheinen.

In diesem Übergang gipfelt auch der gesamte periegetische Duktus, gespickt von zumeist sprechenden Namen auf Brasilianisch und Tupi, angefangen von Orts- und Gebietsbezeichnungen (Toponyme, Oronyme, Hydronyme), über Ethnonyme bis hin zu Anthroponymen und Zoonymen. All diese Begrifflichkeit ist im Nähesprechen durch die changierende Perspektivität Jäger / Jaguar determiniert und bildet somit für den Translationsakt eine umfassende Aufgabe, die über isolierte lexikalische Entscheidungen hinausgeht. So erscheint es beispielsweise arbiträr, wenn in einer Reihe übersetzter Tiernamen kursiv hervorgehoben die "urucuera" (Kaninchenkauz) erscheint. Diesem weit verbreiteten Vogel kommt kein besonderer Nähe-Status $\mathrm{zu}$, seine aus dem Tupi-Guarani stammende Benennung ist in vielen Regionen Brasiliens geläufig. ${ }^{17}$

Der zweite Aspekt betrifft das Astronym "sejuçu” oder "cejuçu”. Es erscheint nicht im Glossar, wohl da es sich bei Erstnennung als "Sejuçu-Gestirn" (Rosa/CMC 34) selbst hinreichend als solches $\mathrm{zu}$ erkennen gibt. Allerdings ist es für den Bedeutungsaufbau des Nähesprechens mehr als ein illustratives Element des Nachthimmels, wie die folgende Passage (mit "Sejucú" graphisch und damit lautlich entstellt) beweist:

Ich kann nicht gefangen sein; meine Mutter hat mir erzählt, daß ich nicht gefangen sein kann, wenn ich gefangen werde, sterbe ich - weil ich in der Zeit der Kälte geboren wurde, zu einer Stunde, wenn der Sejucú mitten in der Höhe des Him-

\footnotetext{
17 "Urucuera", "urucureia" e "urucuriá" vêm do termo guarani para "coruja", urukure'a. E "Uiracuier”, “Uiracuir”, tem origem na junção dos termos tupiguarani para "pássaro" (gwirá) e para "fincar/cavar abrigo" (kuir). (wikipediaEintrag < https://pt.wikipedia.org/wiki/Coruja-buraqueira > Version 9. Juli 2021)
}

Cad. Trad., Florianópolis, v. 41, no 3 p. 395-410, set-dez, 2021. 
mels steht. Schauen Sie: der Sejucú hat vier Sternchen und noch zwei. Gut: sehen Sie den anderen, der fehlt? Sehen Sie ihn nicht? Der andere - das bin ich... (Rosa/CMC 52)

In der für das Tupi charakteristische Zählung bis vier wird ein Sternbild besprochen, das in den europäischen Sprachen als die Plejaden oder Siebengestirn erscheint. In den mythischen TupiNarrativen ist das Erkennen des kleinsten siebten Sterns (der normalerweise nicht mitgezählt wird) mit der Prophezeiung von baldigem Tod - Versetzung an den Nachthimmel - verbunden (Ávila \& Trevisan 308-310). Dies erfüllt sich für das Ich im performativen Ende von Meu tio o Iauaretê.

Ohne dies weiter auszuführen oder gar weitere Beispiele für die hybride sprachliche Setzung Jäger / Jaguar zu bringen, bleibt abschließend festzuhalten, dass es für die Übersetzung besser gewesen wäre, die Tupi-Identität als konstituierend für die inszenierte Nähesprache zu vertiefen, statt sich vorschnell mit seiner Reduzierung zu "Tupi-Mischmasch" (Meyer-Clason 78) im Übergang zur Animalisierung und einer selbstreflexiven bis narzisstischen Meta-Lektüre des "zweisprachige[n] Leser[s]" (!) zufrieden zu geben, die im Grunde auch die Haltung des Übersetzers spiegelt:

Der zweisprachige Leser indes wird sich in seiner Liebe zur fremden Sprache vielleicht durch die Tatsache bestärkt fühlen, daß alles Übersetzen, alles Begreifen des ganz anderen von jener Grenze ausgeht, hinter der sich das Unveräußerliche verbirgt. (Meyer-Clason 80) 


\section{Bibliographie}

Ágel, Vilmos; Hennig, Mathilde. "Theorie des Nähe- und Distanzsprechens. Grammatik aus Nähe und Distanz. Theorie und Praxis am Beispiel von Nähetexten 1650-2000, Ágel, V.; Hennig, M. (Eds.). Tübingen, 2006, pp. 3-31.

Ávila, Marcel Twardowsky; Trevisan, Rodrigo Godinho. "Jaguanhenhém. Um estudo sobre a Linguagem do Iauaretê". Lava. Literaturas da Floresta. Magma, FFLCH - USP, v. 12, n. 2, (2015): 297-335.

Campos, Haroldo de. "A Linguagem do Iauaretê". id., Metalinguagem \& outras metas. São Paulo: Perspectiva, 1992, pp. 57-63. ( $1^{\text {a }}$ ed. 1962)

Chiappini, Lígia. 2a Parte: "Entrevista a Curt Meyer-Clason". Scripta, Belo Horizonte, v. 5, n. 10, (2002): 369-378.

Faria, Johnwill Costa; Hatje-Faggion, Válmi. "O problema da oralidade em três traduções de Of Mice and Men, de John Steinbeck". Cadernos de Tradução, Florianópolis, n. 29 (2012): 53-71.

Grossegesse, Orlando. "Wiedergeburt und Nachschöpfung. João Guimarães Rosa im Diskurs des 'jagunço de Munique"”. Guimarães Rosa und Meyer-Clason: Literatur, Demokratie, saber-conviver, Ottmar Ette \& Paulo Soethe (Eds.). Berlin: De Gruyter (mimesis. Romanische Literaturen der Welt), 2020, pp. 97-117.

Meyer-Clason, Curt. "Einführung". id. (ed.), Die Reiher und andere brasilianische Erzählungen. Tübingen / Basel: Horst Erdmann, 1967, pp. 9-22.

Meyer-Clason, Curt. "Nachwort". Mein Onkel der Jaguar, Rosa, João Guimarães. Übersetzung C. Meyer-Clason, Köln: Kiepenheuer \& Witsch, 1981; Tb KiWi, 1994, pp. 75-80.

Meyer-Clason, Curt. "Nachwort". Tutaméia, Rosa, João Guimarães. Übersetzung C. Meyer-Clason, unter Mitarbeit von Horst Nitschack. Köln: Kiepenheuer \& Witsch, 1994, pp. 259-64. 
Oseki-Dépré, Inês. "O tempo do Iauaretê". Atas do Encontro ABRALIC, (2016): 6640-6650.

Perrone, Charles A. "Notas para facilitar a leitura de 'Meu tio o iauaretê'". Hispania vol. 91, n. 4, Dez. (2008): 765-773.

Rosa, João Guimarães. "Meu tio o Iauaratê". id., Estas estórias. Rio de Janeiro: Nova Fronteira, 1985, pp. 160-198

Rosa, João Guimarães. Mein Onkel der Jaguar. Übersetzung C. Meyer-Clason, Köln: Kiepenheuer \& Witsch, 1981; Tb KiWi, 1994.

Rosa, João Guimarães. Tutaméia. Übersetzung C. Meyer-Clason, unter Mitarbeit von Horst Nitschack. Köln: Kiepenheuer \& Witsch, 1994.

Seidinger, Gilca Machado. "Traduzindo o intraduzível: Tutaméia em alemão". Estudos Lingüísticos, XXXV (2006): 838-844.

Seidinger, Gilca Machado. „Entrevista: Horst Nitschak e a tradução de Tutaméia ao alemão". Itinerários, Araraquara, n. 25 (2007): 225-231.

Tait, Thais Calvi. O jogo entre interpretação e performance em Meu tio o Iauaretê, de Guimarães Rosa. Diss. Mestrado, São Paulo: PUC-SP, 2008.

Zumthor, Paul. Performance, réception, lecture. Longueuil: Éditions du Préambule, 1990.

Recebido em: 18/05/2021

Aceito em: 20/08/2021

Publicado em setembro de 2021

Orlando Grossegesse. E-mail: ogro@elach.uminho.pt. ORCID: https://orcid. org/0000-0001-5466-285X. 